\title{
A Survey on VM Migration and Green Cloud Computing
}

\author{
Anjan Patidar \\ SIRTS Bhopal
}

\author{
Megha Vashishtha \\ SIRTS Bhopal
}

\begin{abstract}
Cloud computing has become a part of everyone's life now. So researchers have immense interest in cloud performance and security. For security and to provide single system image so that user gets an illusion that everything is stored at his own system cloud uses concept of virtualization. For that different virtual machines (VMs) runs on same or different servers. These VMs can be migrated from one server to another. VM migration and power consumption on cloud has been a trending area of research. In this paper a survey in done on VM migration techniques with keeping power consumption in mind. So in this paper green algorithm for VM migration are discussed.
\end{abstract}

\section{Keywords}

Cloud, VM, green algorithm, NP-Hard.

\section{INTRODUCTION}

Cloud computing refers to delivery of computing resources online. Cloud is nothing but collection of computing resources which consist of processors and storage resources. Cloud computing provides many special features as compared to traditional computing methods. Cloud services allow individuals and businesses to use software and hardware that are managed by third parties at remote locations. Examples of cloud services include online file storage, social networking sites, webmail, and online business applications. The cloud computing model allows access to information and computer resources from anywhere that a network connection is available. Cloud computing provides a shared pool of resources, including data storage space, networks, computer processing power, and specialized corporate and user applications.

The characteristics of cloud computing include on-demand self service, broad network access, resource pooling, rapid elasticity and measured service. On-demand self service means that customers (usually organizations) can request and manage their own computing resources. Broad network access allows services to be offered over the Internet or private networks. Pooled resources means that customers draw from a pool of computing resources, usually in remote data centres. Services can be scaled larger or smaller; and use of a service is measured and customers are billed accordingly.

The cloud computing service models are Software as a Service (SaaS), Platform as a Service (PaaS) and Infrastructure as a Service (IaaS). In a Software as a Service model, a pre-made application, along with any required software, operating system, hardware, and network are provided. In PaaS, an operating system, hardware, and network are provided, and the customer installs or develops its own software and applications. The IaaS model provides just the hardware and network; the customer installs or develops its own operating systems, software and applications.

\section{LITERATURE SURVEY}

In [1] An admission control and scheduling mechanism proposes which not only maximizes the resource utilization and profit, but also ensures that the QoS requirements of users are met as specified in SLAs.Mixed Workload Aware Policy (MWAP) is implemented to consider the workload of different types of application such transactional and noninteractive batch jobs. The proposed mechanism provides substantial improvement over static server consolidation and reduces SLA violations.

In [2] In this paper VM consolidation problem which is a NP Hard problem is solved by applying metaheuristic algorithm ACO. The objective is to lower down the energy consumption of the overall algorithm. And the algorithm also reduces VM migrations.

In [3] A novel allocation and selection policy for the dynamic virtual machine (VM) consolidation in virtualized data centers to reduce energy consumption and SLA violation. Firstly, it detect overloading hosts in virtual environments and then apply a method to select VMs from those overloading hosts for migration.VM Provisioning Method to Improve the Profit and SLA Violation of Cloud Service Providers.

In [4] proposed an Threshold based algorithm for VM provisioning among multiple service providers that reduces SLA Violation. It uses two threshold values and two type of VMs (on-demand and reserved),These threshold values will be decided by the cloud federation depending on the environmental conditions like current workload, idle capacity of each cloud provider, etc.

In [5] a power friendly algorithm is proposed. This paper compared live and non live VM migration in terms of power consumption

\section{CLOUD ARCHITECTURE}

Cloud architecture consist of following elements :

- Cloudlet.

- $\quad$ Broker.

- Cloud information service (CIS).

- Datacenter.

Cloudlet

Job that are submitted on cloud are termed as cloudlet. Cloudlet has fixed capacity which is measured in terms of million instructions per second (MIPS).

\section{Broker}

Broker is the agent which is responsible for submitting cloudlet on cloud. It request for resources from CIS and interacts with datacenter.

Cloud information service (CIS)

CIS is responsible for allocating resources to cloudlet. It creates VM for handling cloudlet. These VM are also of fixed 
capacity which is measured in terms of MIPS. VM migration from one server to another is also handled by CIS.

\section{Datacenter}

It is the component of cloud architecture which stores all the data. It consists of several storage elements. And it interacts with CIS and broker.

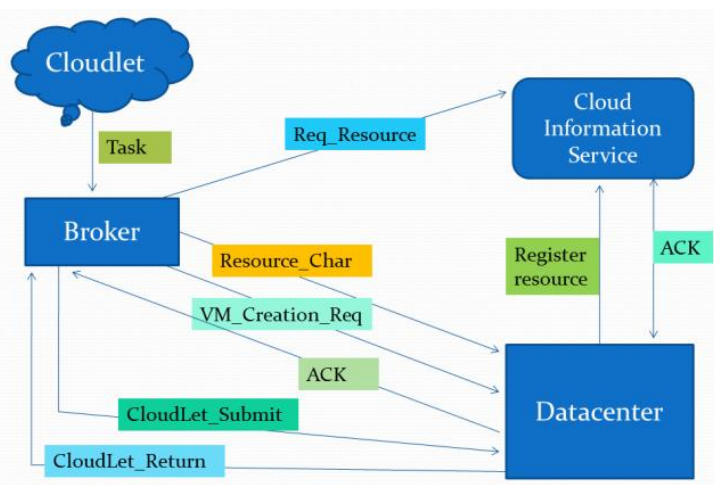

In Cloud task that are termed as cloudlet; are executed over Virtual machines (VM) running on servers. Direct access to server is not given for security reasons. So These VMs runs on servers which act as host to user as they provide services.

\section{VM Migration}

When this VM is moved from one host to other host depending on some criteria is called VM Migration.

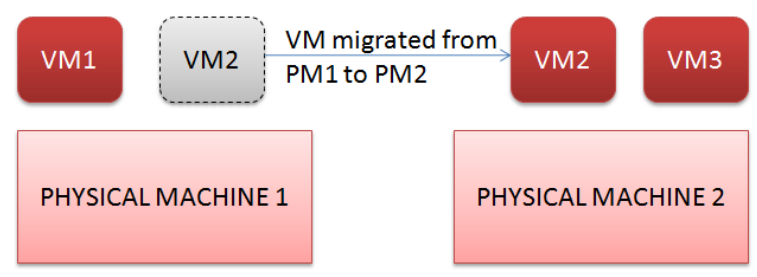

\section{VM Migration techniques}

Following are the VM Migration techniques :

- $\quad$ Energy based VM migration.

- Load based VM migration.

- $\quad$ Fault based VM Migration.

\section{Energy based VM migration}

In this migration technique those Physical machines (PM) which are underloaded. VMs from those machines are migrated to another PM.

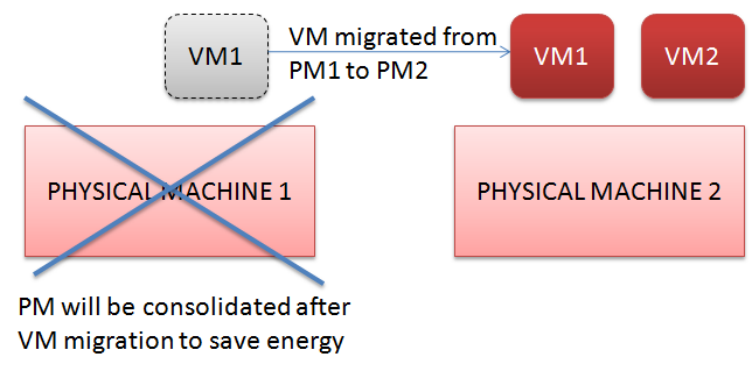

\section{Load based VM migration}

This technique is used to balance load among physical machines (PMs). VMs are migrated from overloaded PM to underloaded PM or Pm which is under normal category after VM migration

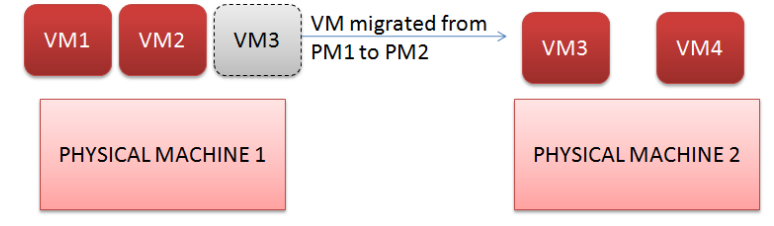

Fault based VM Migration

In this technique whenever any physical machine fails or fault occurs in PM all its VM are migrated to another PM.

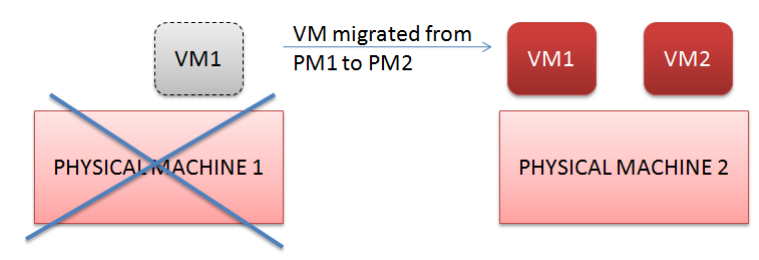

\section{CONCLUSION}

In this paper different VM migration techniques are studied and compared with each other. So it is concluded that energy based VM migration results in power efficient algorithm and comes under green algorithm. Load Based VM migration is to balance load among PMs.

\section{REFERENCES}

[1] Saurabh Kumar Garg, Adel NadjaranToosi, Srinivasa K. Gopalaiyengar, RajkumarBuyya, "SLA-based virtual machine management for heterogeneous workloads in a cloud datacenter," Journal of Network and Computer Applications 1 August 2014.

[2] FahimehFarahnakian, Adnan Ashraf, TapioPahikkala,PasiLiljeberg, JuhaPlosila, Ivan Porres, and HannuTenhunen "Using Ant Colony System to ConsolidateVMs for Green Cloud Computing" in IEEE TRANSACTIONS ON SERVICES COMPUTING, VOL. 8, NO. 2, MARCH/APRIL 2015.

[3] Zhibo Cao and Shoubin Dong, "Dynamic VM consolidation for energy-aware and SLA violation reduction in cloud computing," 13th International Conference on Parallel and Distributed Computing, Applications and Technologies 2012.

[4] Komal Singh Patel and A. K. Sarje, "VM Provisioning Method to Improve the Profit and SLA Violation of Cloud Service Providers," IEEE International Conference, Cloud Computing in Emerging Markets (CCEM) 11-12 Oct. 2012 .

[5] David Aikema, AndreyMir+tchovski, Cameron Kiddle, and Rob Simmonds "Green Cloud VM Migration: Power Use Analysis"

[6] Komal Singh Patel and A. K. Sarje, "VM Provisioning Method to Improve the Profit and SLA Violation of Cloud Service Providers," IEEE International Conference, Cloud Computing in Emerging Markets (CCEM) 11-12 Oct. 2012.

[7] K. S. Patel and A.K. Sarje, "VM Provisioning Policies to Improve the Profit of Cloud Infrastructure Service Providers," ICCCNT-12, July.2012.

[8] Gundeep Singh Bindra, Prashant Kumar Singh,Seema Khanna,Krishen Kant Kandwal, "Cloud Security : Analysis and Risk Management of VM Images," 
Proceeding of IEEE International Conference on Information and Automation Shenyang, China, June 2012.

[9] Eeraj Jan Qaisar, "Introduction to Cloud Computing for Developers,” In IEEE C2012.

[10] A. Beloglazov, R. Buyya, "Optimal Online Deterministic Algorithms and Adaptive Heuristics for Energy and Performance Efficient Dynamic Consolidation of Virtual Machines in Cloud Data Centers," Concurrency and Computation: Practice and Experience (CCPE), Wiley
Press, New York, USA, Sep. 2012, pp. 1397-1420, doi: 10.1002/cpe.1867.

[11] Zhibo Cao and ShoubinDong , "Dynamic VM consolidation for energy-aware and SLA violation reduction in cloud computing," 13th International Conference on Parallel and Distributed Computing, Applications and Technologies 2012.

[12] YonggenGu, Wei Zhang, YonggenGu, Jie Tao, "A Study of SLA Violation Compensation Mechanism in Complex Cloud Computing Environment,” In IEEE (C) 2012. 